\title{
Behavior, Fate, and Mass Loading of Short Chain Chlorinated Paraffins in an Advanced Municipal Sewage Treatment Plant
}

\author{
Lixi Zeng, ${ }^{\dagger \dagger}$ Huijuan $\mathrm{Li}^{\dagger}{ }^{\dagger}$ Thanh Wang, ${ }^{\dagger}$ Yan Gao, ${ }^{\dagger}$ Ke Xiao, ${ }^{\dagger}$ Yuguo Du, ${ }^{\dagger \neq}$ Yawei Wang, ${ }^{* \dagger}$ \\ and Guibin Jiang ${ }^{\dagger}$
}

${ }^{\dagger}$ State Key Laboratory of Environmental Chemistry and Ecotoxicology, Research Center for Eco-Environmental Sciences, Chinese
Academy of Sciences, Beijing 100085, China
${ }^{\ddagger}$ School of Chemistry and Chemical Engineering, University of Chinese Academy of Sciences, Beijing 100049, China

Supporting Information

ABSTRACT: Sewage treatment plants (STP) are an important source of short chain chlorinated paraffins (SCCPs) to the ambient environment through discharge of effluent and application of sludge. In this work, a field study was conducted to determine the behavior and possible removal of SCCPs during the sewage treatment process in an advanced municipal STP in Beijing, China. SCCPs were detected in all sewage water and sludge samples, and $97 \%$ of the initial mass loading in raw sewage was found to be associated with suspended matter. The total concentrations in raw influent, tertiary effluent, and dewatered sludge were $184 \pm 19 \mathrm{ng} / \mathrm{L}, 27 \pm 6 \mathrm{ng} / \mathrm{L}$, and $15.6 \pm 1.4 \mu \mathrm{g} / \mathrm{g}$ dry weight (d.w.), respectively. The dissolved concentrations of total SCCPs ( $\sum$ SCCPs) significantly decreased during mechanical, biological, and chemical treatments. SCCP homologue profiles in aqueous phase were distinctly different from those in solid phase. Along the treatment

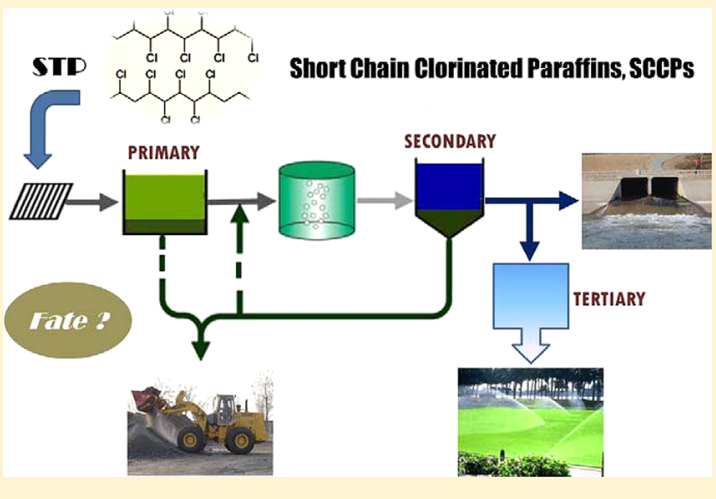
process, the relative abundance of shorter chain and lower chlorinated congeners gradually increased in sewage water, but no obvious variations of homologue profiles were found in sludge. Mass flow analysis indicated, the removal efficiency in aqueous phase for $\sum$ SCCPs was $82.2 \%$, and the congener-specific removal efficiencies were positively related to their solid-water partition coefficients $\left(K_{\mathrm{d}}\right)$. Mass balance results indicated that $0.8 \%$ and $72.6 \%$ of the initial SCCP mass loading were ultimately found in the effluents and dewatered sludge, respectively, while the remaining $26.6 \%$ was lost mainly due to biodegradation/ biotransformation. It was suggested that the activated sludge system including basic anaerobic-anoxic-aerobic processes played an effective role in removing SCCPs from the wastewater, while the sorption to sludge by hydrophobic interactions was an important fate of SCCPs during the sewage treatment.

\section{INTRODUCTION}

Chlorinated paraffins (CPs) are a complex mixture of polychlorinated $n$-alkanes with chlorination degree from 30 to 70\%. ${ }^{1}$ CPs are subdivided into short (SCCPs, C10-13), medium (MCCPs, C14-17), and long chain CPs (LCCPs, C18-30) according to their carbon chain length. ${ }^{2}$ Over $200 \mathrm{CP}$ formulations are in use for a wide range of industrial applications, such as flame retardants and plasticizers, as additives in metal working fluids, sealants, paints, and coatings, and as solvent for Dichloramine $\mathrm{T}$ (germicide). ${ }^{3}$ The presence of CPs in the environment has been ascertained in various environmental matrices (air, water, soil, sediment, and biota) worldwide. $^{4-9}$ Compared to other two groups of CP mixtures, SCCPs have received growing global attention in recent years for their long-range transport, ${ }^{10,11}$ persistence in the environment, ${ }^{12}$ bioaccumulation in food webs, ${ }^{13}$ and potential toxicity to aquatic organisms. ${ }^{14,15}$ Currently, SCCPs are being reviewed by Stockholm Convention's POP Review Committee (POPRC) to evaluate whether this substance meet the criteria on persistent organic pollutants (POPs). ${ }^{16}$ However, to date, very limited data for environmental fate and toxicology of SCCPs are available as a result of the complexity of their compositions and the challenge for quantifying these compounds. $^{17,18}$

The releases of SCCPs into the environment can occur during production, storage, transportation, industrial usage of manufactured products, disposal and burning of waste, etc. ${ }^{19}$ The possible sources of releases to water are mainly from industrial effluents, consumer usage of SCCP-containing products, and leaching and runoff from landfill and waste disposal sites. ${ }^{16}$ Of these, some of the released SCCPs are collected in sewer systems and ultimately accumulate into sewage treatment plants (STPs). ${ }^{20}$ Generally, STPs play an important role in removing many contaminants from wastewater. ${ }^{21}$ They are normally designed to eliminate solids,

Received: October 17, 2012

Revised: December 14, 2012

Accepted: December 19, 2012

Published: December 19, 2012 


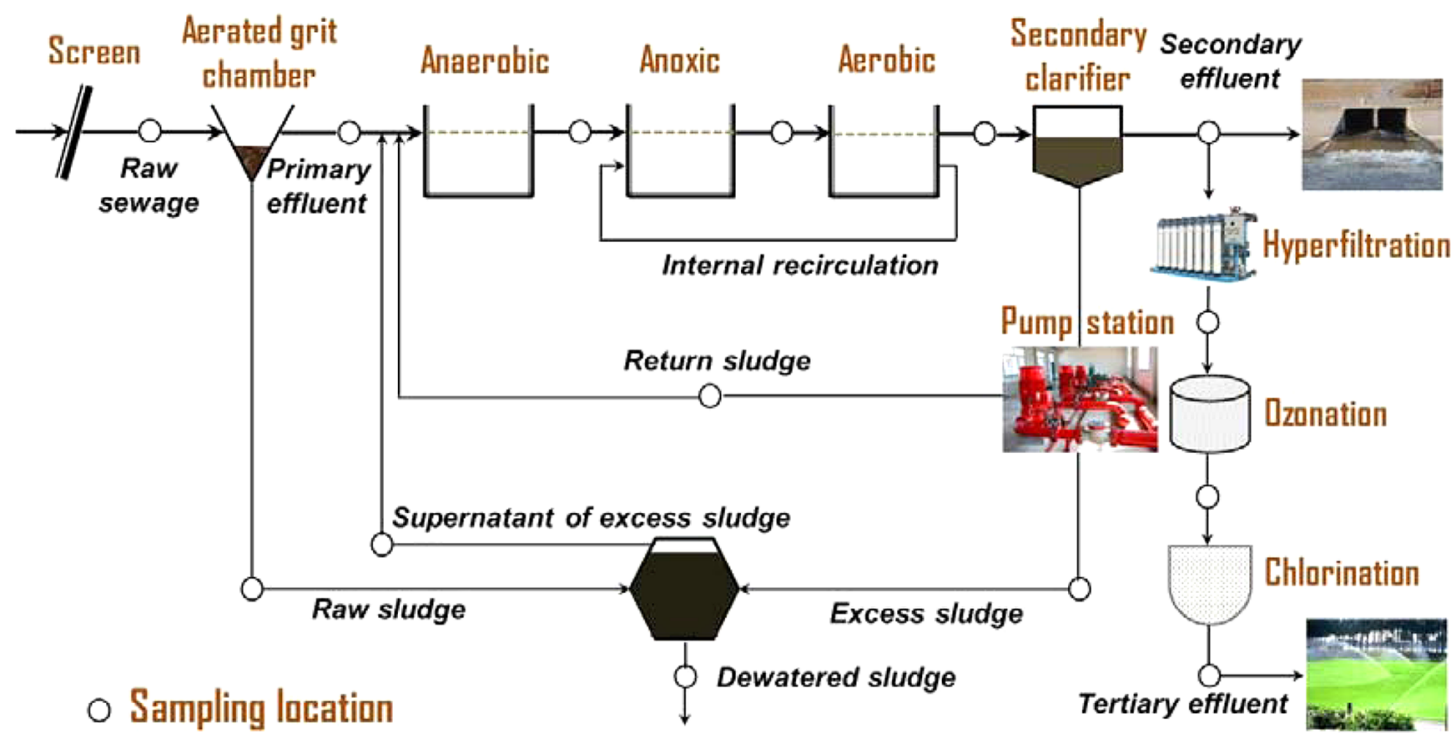

Figure 1. Flow scheme of the investigated municipal STP and sampling locations.

particulate contaminants, nutrients, and dissolved biodegrable organic matter but not specifically designed for the removal of the emerging contaminants such as SCCPs, ${ }^{22-24}$ and what is the fate and major removal units of these compounds still remains unknown.

There are only very scarce data available at present on the occurrence and levels of SCCPs in the STPs. Stevens et al. ${ }^{25}$ investigated the occurrence of CPs in the digested sludge from 14 U.K. STPs, with the total concentrations of SCCPs and MCCPs ranging from 7 to $200 \mu \mathrm{g} / \mathrm{g}$ d.w. and from 30 to 9700 $\mu \mathrm{g} / \mathrm{g}$ d.w., respectively. Iino et al. $^{26}$ reported the total concentrations of SCCPs ranging from 220 to $360 \mathrm{ng} / \mathrm{L}$ in influents and 16 to $35 \mathrm{ng} / \mathrm{L}$ in effluents from three STPs in Japan. Our recent study found significantly high concentrations of SCCPs $(0.8-52.7 \mu \mathrm{g} / \mathrm{g}$ d.w. $)$ in 52 sewage sludge from all surveyed STPs in China, ${ }^{20}$ and the effluents discharged from a municipal STP contain high residual levels of SCCPs. ${ }^{27}$ The treated effluents as irrigation water source for farmland have been proved to be responsible for the accumulation of SCCPs in soils. ${ }^{28}$ All these results demonstrated that the release of effluent and sludge from STPs is an important source of SCCP contamination to the environment. Therefore, it is very essential to understand the removal and behavior of SCCPs in the STPs in order to reduce their ecological risk and potential negative impacts on the environment.

In the present study, sewage water and sludge samples along the whole sewage treatment process were collected in an advanced municipal STP with anaerobic/anoxic/aerobic (A/A/ $\mathrm{O})$ bioreactors as the core treatment units. We carried out a detailed examination of SCCP concentrations and homologue patterns, and the key factors affecting the fate and transport of SCCPs during sewage treatment process. A mass balance analysis was applied to establish mass flow in the STP and removal mechanisms of these compounds inside the treatment units. The results of this study are expected to gain insights into the behavior, fate, and transport of SCCPs in the typical municipal STP.

\section{MATERIALS AND METHODS}

Flow Scheme of Sewage Treatment Plant. The STP selected serves a residential population of about 814000 in
Beijing, China, and has four parallel activated sludge-type secondary treatment systems, with a total sewage treatment capability of $400000 \mathrm{~m}^{3} /$ day. The flow scheme of the STP is illustrated in Figure 1. The influents consists mainly of domestic wastewater, which first passes through the mechanical screen and aerated grit chamber as the primary clarification. Then, the primary effluent flows directly through an activated sludge system, which is comprised of the anaerobic/anoxic/ aerobic $(\mathrm{A} / \mathrm{A} / \mathrm{O})$ bioreactors. After clarification in the secondary sedimentation tank, $60 \%$ of the second effluent is directly discharged to a receiving river, while the remaining $40 \%$ of the second effluent continuously passes to a tertiary treatment system as the advanced treatment, which is comprised of three physicochemical steps including hyperfiltration, ozonation, and chlorination processes. The final tertiary effluent is used for irrigation water. The hydraulic retention time (HRT) in the aerated grit chamber, anaerobic tank, anoxic tank, aerobic tank, and secondary sedimentation tank were $15,2,3,11$, and $8 \mathrm{~h}$, respectively, and the total solid retention time was 20-25 day. Most of activated sludge in the secondary sedimentation tank was returned to the anaerobic bioreactor, and the rest was pumped into a storage tank as excess sludge. After being dewatered, the excess sludge was carried away for final disposal.

Sample Collection and Pretreatment. Three sets of samples along sewage treatment process were collected on three separate days under normal dry weather conditions, spaced over a two week period between the end of June and early August 2012 at the STP. The sampling locations in the STP are also shown in Figure 1. During the sampling period, the sewage influent to the STP kept around $200000 \mathrm{~m}^{3} / \mathrm{d}$, and the water temperatures were $23-25{ }^{\circ} \mathrm{C}$ in biological treatment units. The water flow at each treatment unit and several regularly measured parameters (e.g., BOD5, COD, DO, NH4$\mathrm{N}$, and $\mathrm{NO} 3-\mathrm{N}$ ) are shown in the Supporting Information, Table S1. The average removal efficiencies of BOD5, COD, total phosphate, and suspended solid ranged from $91 \%$ to $98 \%$.

All samples were taken at the outlet of every treatment step. Two sampling methods were selected to compare the variations of SCCPs in raw influents in different stages of a day. The first and second sets of samples were taken as grab samples between 
2 p.m. and 6 p.m. during two sampling days. The third set of samples were collected as composite samples. The timeproportional $24 \mathrm{~h}$ composite samples of raw sewage and primary, secondary, and tertiary effluents were collected by using automatic samplers with sampling interval of $2 \mathrm{~h}$. Other sludge-liquid and sludge samples were obtained as timeproportional $24 \mathrm{~h}$ composite samples by combining four grab samples collected four times a day with sampling interval of $6 \mathrm{~h}$. All samples, except dewatered sludge, were immediately centrifuged to separate into the aqueous phase and solid phase after sampling. Water samples were filtered through a glass microfiber filter GF/C $1.2 \mathrm{~mm}$ (Whatman, Maidstone, U.K.) and stored at $4{ }^{\circ} \mathrm{C}$ in refrigerator prior to analysis. Sludge samples were frozen within hours in refrigerator and further freeze-dried in a freeze-dryer, then homogenized using an analytical grinding mill, and sifted through a sieve with 100 meshes, and stored at $-20{ }^{\circ} \mathrm{C}$ in refrigerator until analysis. Details about sample extraction and cleanup procedures of SCCPs are given in the Supporting Information.

Instrumental Analysis, Identification, and Quantification. Instrumental analysis was performed using a highresolution gas chromatograph coupled with electron capture negative ion-low resolution mass spectrometer (HRGC/ECNILRMS, Agilent, U.S.A.). The two most abundant isotopes of the $[\mathrm{M}-\mathrm{Cl}]^{-}$cluster for individual $\mathrm{CP}$ congener groups with 5-10 chlorine atoms were monitored for quantification and qualification described by Tomy et al. ${ }^{29-31}$ To identify the potential interferences and ensure high instrumental sensitivity, SCCP and MCCP congeners were simultaneously monitored by four individual injections in eight retention time windows. Detailed information on instrumental analysis can be found in our previous work. ${ }^{28}$

Different SCCP congener groups were identified by monitoring selected $[\mathrm{M}-\mathrm{Cl}]^{-}$ions, comparing retention time, chromatographic peak shapes, and isotope ratio with those of standards. $^{32}$ The interferences of MCCP congeners are effectively mitigated by mathematical calculations described in our previous works based on theoretical natural isotopic abundances of congeners. ${ }^{28}$ Quantification of SCCPs was based on the procedure described by Reth et al. ${ }^{33}$ to compensate the influence of chlorine contents on the total response factors between environmental samples and SCCP standards. A significant linear correlation was obtained between the total response factors of SCCP standards and chlorine contents $\left(R^{2}\right.$ $=0.978)$.

Mass Balance Calculations. Mass balance was calculated by multiplying concentrations of SCCPs by average daily flow rates. $^{34}$

$$
W=C_{\text {dissolved }} Q+C_{\text {adsorbed }} Q C_{\mathrm{TSS}}
$$

where $W$ is the total mass flow of SCCPs dissolved in water and adsorbed to sludge $(\mathrm{g} / \mathrm{d}) ; C_{\text {dissolved }}$ and $C_{\text {adsorbed }}$ represent the dissolved $(\mathrm{ng} / \mathrm{L})$ and adsorbed $(\mu \mathrm{g} / \mathrm{g})$ concentrations, respectively; $Q$ is water flow $\left(\mathrm{m}^{3} / \mathrm{d}\right)$; and $C_{\mathrm{TSS}}$ represents the content of total suspended solids $(\mathrm{mg} / \mathrm{L})$.

To assess the contributions of sorption and degradation of SCCPs in the STP, the mass loading of SCCPs that was lost from total degradation or transformation during sewage treatment process was calculated using the following equation: ${ }^{35}$

$$
W_{\text {lost }}=W_{\text {influent }}-W_{\text {effluent }}-W_{\text {sludge }}
$$

where $W_{\text {influent }}$ and $W_{\text {efluent }}$ represent the mass loading of SCCPs in influent and effluent $(\mathrm{g} / \mathrm{d})$, respectively; $W_{\text {sludge }}$ is the mass output in dehydrated sludge $(\mathrm{g} / \mathrm{d})$.

To assess the mass variations of SCCPs in individual treatment stages and the whole treatment process, the mass change percentage (removal efficiency) was calculated using $\left(W_{\text {inflow }}-W_{\text {outflow }}\right) / W_{\text {inflow }} \times 100 \%$, where $W_{\text {inflow }}$ and $W_{\text {outflow }}$ represent the mass loading of SCCPs in the inflow and outflow of individual treatment unit, respectively.

\section{RESULTS AND DISCUSSION}

Concentrations of SCCPs in Sewage Water and Sludge along Treatment Process. SCCPs were detected in both sewage water (dissolved/aqueous phase, $\mathrm{ng} / \mathrm{L}$ ) and sludge samples (adsorbed/solid phase, $\mu \mathrm{g} / \mathrm{g}$ d.w.) at each treatment step in the STP. The measured concentrations of four carbon chain groups $(\mathrm{C} 10, \mathrm{C} 11, \mathrm{C} 12, \mathrm{C} 13)$ and total SCCPs ( $\sum$ SCCPs) along the whole treatment process were shown in Table 1. The detailed concentrations of six SCCP homologues with 5-10 chlorine atoms were also listed in Supporting Information Table S2. In the analysis of water and sludge samples, no systematic difference could be detected in the measured concentrations during the three sampling days. The average of the concentrations from each treatment step was therefore calculated and used for the mass flow analysis. Statistical analysis of significance test between the mean concentrations for SCCPs was conducted using one-sided paired $t$-test at a $10 \%$ level of significance $(\alpha=0.1)$. The dissolved concentrations between the adjacent treatment steps were identified to be significantly different, meanwhile, the adsorbed concentrations of primary sludge vs activated sludge and activated sludge vs dewatered sludge were also identified to be significantly different.

SCCPs were found at quantifiable concentrations in all sewage water samples from raw influent to final treated effluent. Of all carbon chain groups, the shortest chain homologue of $\mathrm{C} 10$ showed the highest dissolved concentrations, followed by C11, C12, and C13. Overall, $\sum$ SCCPs in aqueous phase decreased significantly along the treatment processes in the STP, which were shown in the Supporting Information, Figure S1. The dissolved concentrations of $\sum$ SCCPs in raw influent were observed at $184 \pm 19 \mathrm{ng} / \mathrm{L}$, with small fluctuation during the three sampling campaigns, which were comparable to those reported by Iino et al. ${ }^{26}$ in Japan but about 20 -fold lower than our previous finding in influent of another STP receiving 20\% industrial wastewater. The dissolved $\sum$ SCCPs concentration in primary effluent was found to decrease by about $16 \%$, with 154 $\pm 14 \mathrm{ng} / \mathrm{L}$. The decreasing concentration was possibly due to sorption of SCCPs onto inorganic particles and small degradation during the long hydraulic retention time at aerated grit chamber. The great majority of SCCPs in raw sewage passed to the secondary treatment system, and the dissolved $\sum$ SCCPs continuously decreased to $96 \pm 5,65 \pm 4,52 \pm 9 \mathrm{ng} /$ $\mathrm{L}$ in the anaerobic, anoxic, aerobic bioreactors, and $37 \pm 3 \mathrm{ng} / \mathrm{L}$ in secondary sedimentation tank, respectively. Comparing to the initial dissolved concentrations in raw influent (100\%), the decreasing concentration percentages along the secondary treatment processes were $48.0 \%, 64.7 \%, 71.6 \%$, and $80.0 \%$, respectively. It was noted that the concentrations of dissolved SCCPs in secondary effluent were obviously lower than those in aerobic effluent, implied that sorption of SCCPs onto activated sludge and/or constant degradation might occur in secondary sedimentation tank. The results also indicated that 


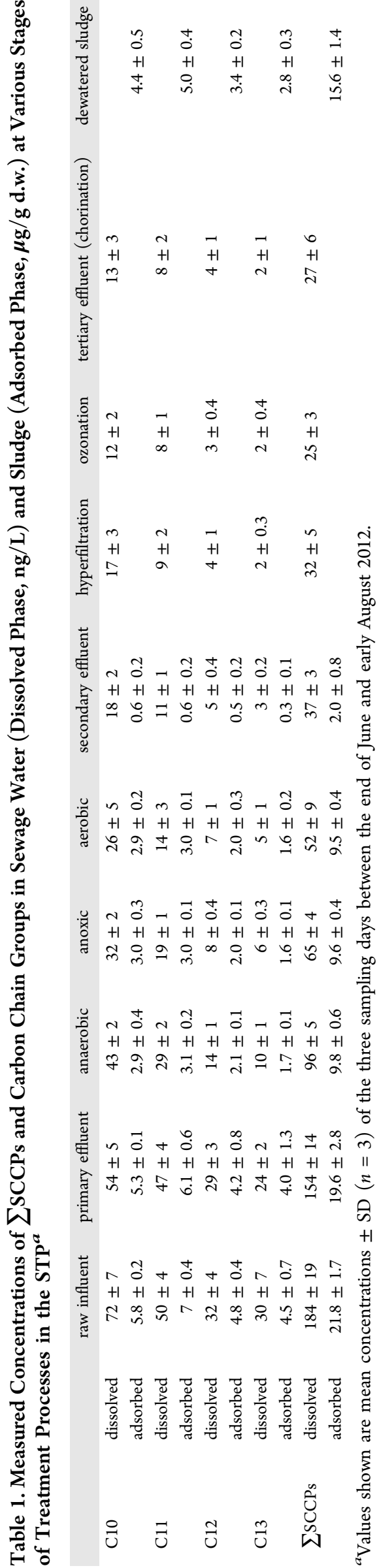

the secondary treatment is the most important process for removing SCCPs in aqueous phase in the STP. In the tertiary treatment steps, $\sum$ SCCPs further decreased, and some congeners with 10 chorines were under the limit of detection, but only a small fraction of SCCPs was reduced by the ultrafiltration and ozonation steps. Chlorination did not appear to the efficacy on additional reduction of SCCP levels. The dissolved concentrations of $\sum$ SCCPs in final tertiary effluent was $27 \pm 6 \mathrm{ng} / \mathrm{L}$, which accounted for about $15 \%$ of the initial concentration in raw sewage.

As shown in Table 1, SCCPs were detected in all suspended particles and sludge samples with very high adsorbed concentrations. Among four carbon chain groups, the C11 homologue exhibited the highest concentration in all solid samples, followed by $\mathrm{C} 10, \mathrm{C} 12$, and $\mathrm{C} 13$ homologues. The suspended particles in raw sewage and primary effluent showed the highest levels of SCCPs, and the total adsorbed concentrations were up to $21.8 \pm 1.7 \mu \mathrm{g} / \mathrm{g}$ and $19.6 \pm 2.8$ $\mu \mathrm{g} / \mathrm{g}$ d.w., respectively, which were about 2 -fold higher than those of activated sludge in bioreactors. Based on the measured content of total suspended particles $(282 \mathrm{mg} / \mathrm{L})$ in raw sewage, the absolute amounts of adsorbed SCCPs in suspended particles were 30 times higher than those of dissolved SCCPs in aqueous phase. The results indicated that SCCPs prefer to adsorb onto suspended particles/sediments than dissolve in aqueous phase due to their high hydrophobicity (log $K_{\mathrm{ow}}$ range $4.1-11.3) .{ }^{36}$ Because of a lack of a primary sedimentation tank and because the aerated grit chamber can only remove the grits rather than organic suspended particles, most of the adsorbed SCCPs in raw sewage entered primary effluent and passed through the secondary treatment system. The adsorbed concentrations of SCCPs in activated sludge along anaerobic/ anoxic/aerobic (A/A/O) treatment units showed a slight decrease trend, which was from $9.8 \pm 0.6 \mu \mathrm{g} / \mathrm{g}$ d.w. to $9.5 \pm 0.4$ $\mu \mathrm{g} / \mathrm{g}$ d.w. Both return sludge and excess sludge also showed a comparable concentration to activated sludge in aerobic tank. The measured concentrations of SCCPs in various types of sludge samples were similar, mainly because a large amount of activated sludge was constantly cycled as internal recirculation and return sludge in the STP. The low degradation rate relative to large mass loading in sludge may be another important factor. The concentrations of SCCPs in dewatered sludge were $15.6 \pm 1.4 \mu \mathrm{g} / \mathrm{g}$ d.w., which were much higher than those in activated sludge but in the medial range of our previous reported levels. ${ }^{20}$

Variations of Congener Group Patterns along Treatment Process and the Implications for Potential Degradation Pathway. To ascertain the relative abundance variations of SCCP homologues and understand the potential degradation along the sewage treatment process, SCCP homologue profiles categorized by carbon and chlorine atom groups in sewage water and sludge samples at different treatment stages were plotted in Figure 2. The individual congener group abundance profiles in primary, secondary, anaerobic, and tertiary effluents were presented in the Supporting Information, Figure S2. By contrast with those in sewage water, the individual congener groups abundance profiles in primary, secondary, anaerobic, and dewatered sludge were also illustrated in the Supporting Information, Figure S3.

Significant differences in SCCP homologue profiles could be found in the aqueous phase at different treatment stages (Figure 2). In the carbon homologue profile, the shortest chain $\mathrm{C} 10$ were predominant congeners in all sewage water samples, 

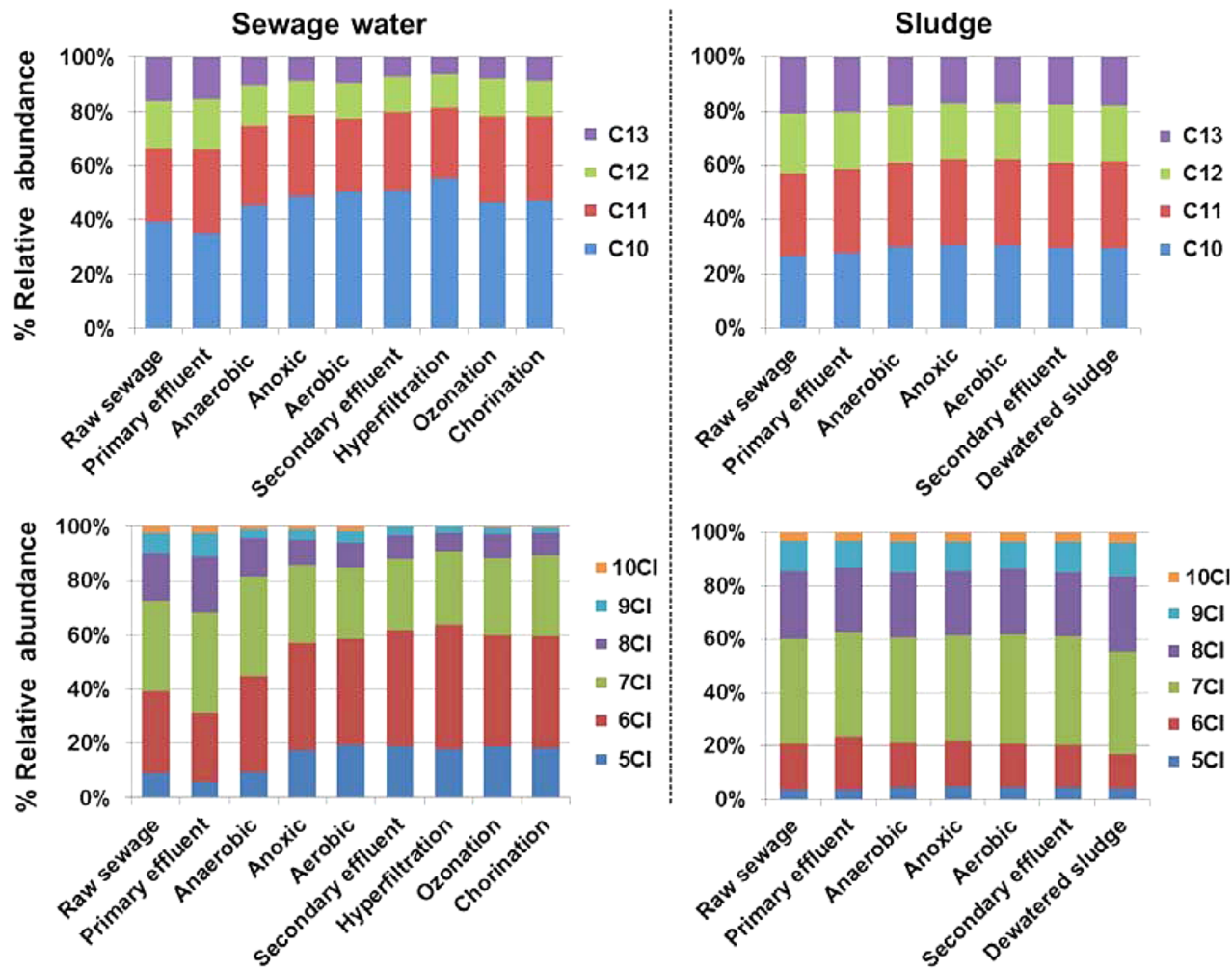

Figure 2. Variations of relative abundance of SCCP congener groups categorized by carbon and chlorine atoms in sewage water (dissolved phase) and sludge (adsorbed phase) along treatment processes of the STP.

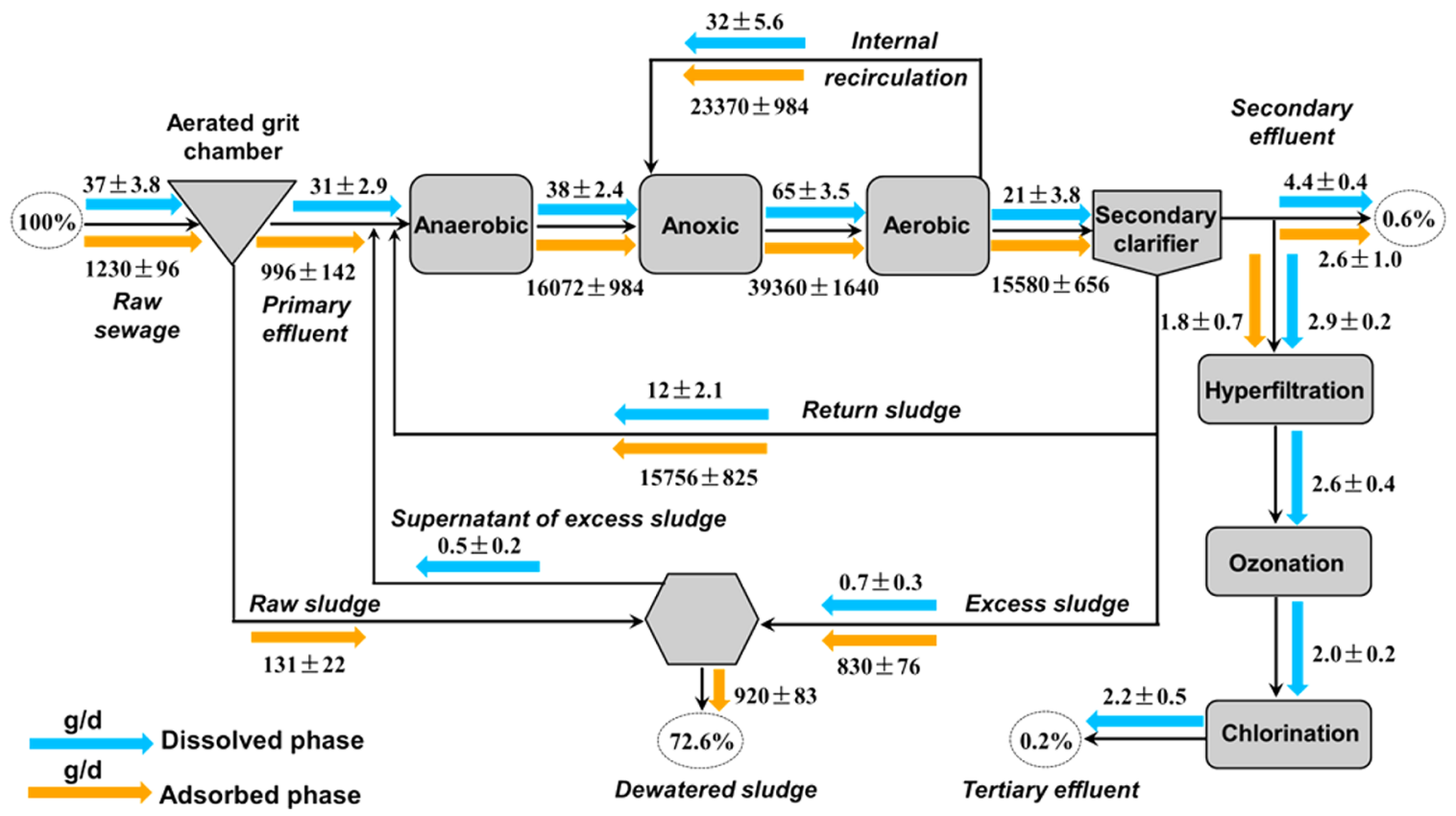

Figure 3. Total mass flows $(\mathrm{g} / \mathrm{d})$ of detected $\sum$ SCCPs in sewage treatment processes and the mass fraction emitted by the plant in effluents and dewatered sludge relative to the total initial mass loading $(100 \%)$ of raw sewage.

followed by C11, C12, and C13 homologues. This might be due to shorter chain congeners having higher water solubility and lower $K_{\mathrm{ow}}$ value, and thus, they are more easily partitioned into the aqueous phase. We also found that the relative abundance of shorter carbon chain groups gradually increased along the whole treatment process. The relative abundance for $\mathrm{C} 10, \mathrm{C} 11, \mathrm{C} 12$, and $\mathrm{C} 13$ homologues were $39.1 \%, 27.2 \%$,
$17.2 \%$, and $16.5 \%$ in raw sewage and $50.8 \%, 29.0 \%, 13.1 \%$, and $7.1 \%$ in secondary effluent, respectively. In the chlorine homologue profile, for raw sewage and primary effluent, the most and second most abundant chlorine congener groups were $7 \mathrm{Cl}$ and $6 \mathrm{Cl}$, followed by $8 \mathrm{Cl}$. However, after aerobic treatment, the most and second most dominant chlorine congener groups changed to $6 \mathrm{Cl}$ and $7 \mathrm{Cl}$, followed by $5 \mathrm{Cl}$. The 


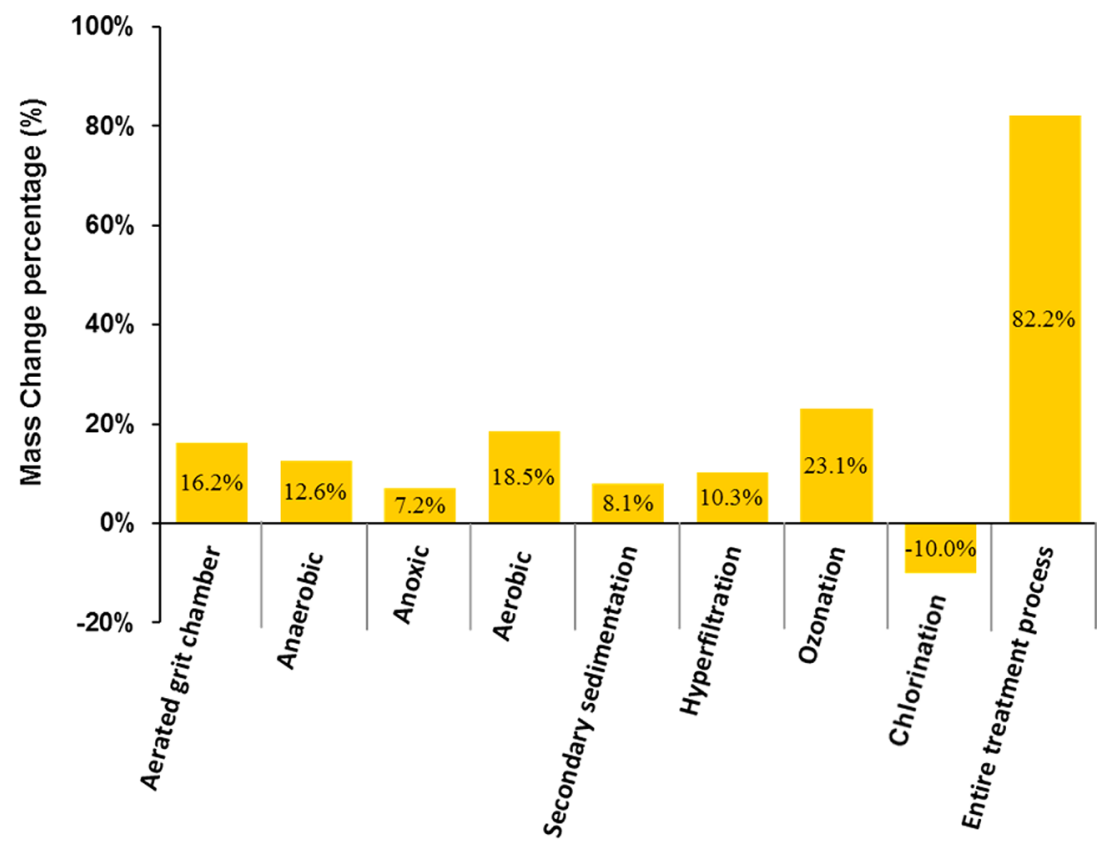

Figure 4. Mass change percentages of $\sum$ SCCPs in aqueous phase at different treatment units based on the mass loading in the inflow and outflow of individual treatment unit, and total mass change percentage (removal efficiency) in aqueous phase during the entire treatment process based on the mass loading in the raw sewage and effluents.

increasing abundance trend toward lower chlorinated congeners along the treatment process was more significant than shorter chain congeners. Compared to other halogenated contaminations such as PBDEs, congener distribution patterns with halogenations were found to be similar at various stages of wastewater treatment process. ${ }^{21}$ These results indicated biological and chemical treatments may play an important role on the composition distribution of SCCPs in aqueous phase.

No significant variations in SCCP homologue profiles in solid phase were found throughout the treatment process because of continuous cycle of activated sludge in the STP, but congener distribution patterns were distinctly different from those in aqueous phase (Figure 2). In the carbon homologue profile, SCCPs in all sewage sludge were predominated by $\mathrm{C} 11$ homologue, followed by $\mathrm{C} 10, \mathrm{C} 12$, and $\mathrm{C} 13$ homologues. There was a slight increasing trend toward shorter chain congeners along the treatment process in solid phase, which was consistent with the variation in aqueous phase. The relative abundance for $\mathrm{C} 10, \mathrm{C} 11, \mathrm{C} 12$, and $\mathrm{C} 13$ homologues were $26.5 \%, 30.7 \%, 22.1 \%$, and $20.7 \%$ in raw sludge, while changed to $29.8 \%, 31.7 \%, 20.8 \%$, and $17.7 \%$ in dewatered sludge. Almost no discernible changes could be found in the chlorine homologue profiles of all sludge samples except dewatered sludge, which contained more heavier chorine-substituted congeners such as $8 \mathrm{Cl}$ and $9 \mathrm{Cl}$. It was noted that, in all sludge samples, the most and second most abundant chlorine congener groups were $7 \mathrm{Cl}$ and $8 \mathrm{Cl}$, followed by $6 \mathrm{Cl}$, which were different from those in both raw sewage and other treated effluents.

The variation of SCCP homologue profiles in sewage water and sludge at various treatment stages are probably related to their different degradation capacities and/or adsorption behaviors. On the basis of the results described, long carbon chain congeners would be more easily adsorbed onto activated sludge and then susceptible to degradation. Besides, dechlorination of higher chlorinated to lower chlorinated congeners by activated sludge during wastewater treatment process might take place.

Mass Flow Analysis for Behavior of SCCPs during Wastewater Treatment. To better understand the partitioning, mass loading, degradation/transformation behavior of SCCPs in the STP, the calculated mass flows of $\sum$ SCCPs in aqueous and solid phases along the entire sewage treatment process were illustrated in Figure 3. The individual mass flows of each SCCP congener group were also reported in the Supporting Information, Table S3. The mass change percentages in the four basic treatment units and three advanced treatment units were analyzed and shown in Figure 4, to assess the major removal units and potential removal mechanism in the STP.

In raw sewage, the combined aqueous and solid phase mass flows of SCCPs were about $1267 \mathrm{~g} / \mathrm{d}$ in the STP, and the mass flows entering the STP were $37 \pm 3.8$ and $1230 \pm 96 \mathrm{~g} / \mathrm{d}$ in the dissolved and adsorbed fraction, respectively. The greater affinity of SCCPs for suspended solids is evident from the fact that $97 \%$ of total SCCP mass flow in raw sewage is associated with particulate matter. The total mass flow was reduced by around 19\% across the aerated grit chamber and about $1027 \mathrm{~g} /$ $\mathrm{d}$ remained in primary effluent entering secondary activated sludge system. The mass change percentages in both aqueous and solid phases were almost equivalent in the aerated grit chamber.

In the activated sludge system, the mass flows in both aqueous and solid phases through the anaerobic and anoxic tanks were obviously increasing. It was worth noting that the total mass of SCCPs in activated sludge was much higher than loading in suspended particles from primary effluent. The increasing mass flows of SCCPs in anaerobic and anoxic tanks mainly originated from large amount recycling of activated sludge with high mass loading of SCCPs from the external and internal recirculation. Subsequently, the mass flows began to significantly decrease across the aerobic tank. During the secondary sewage treatment, the aqueous-phase mass change 


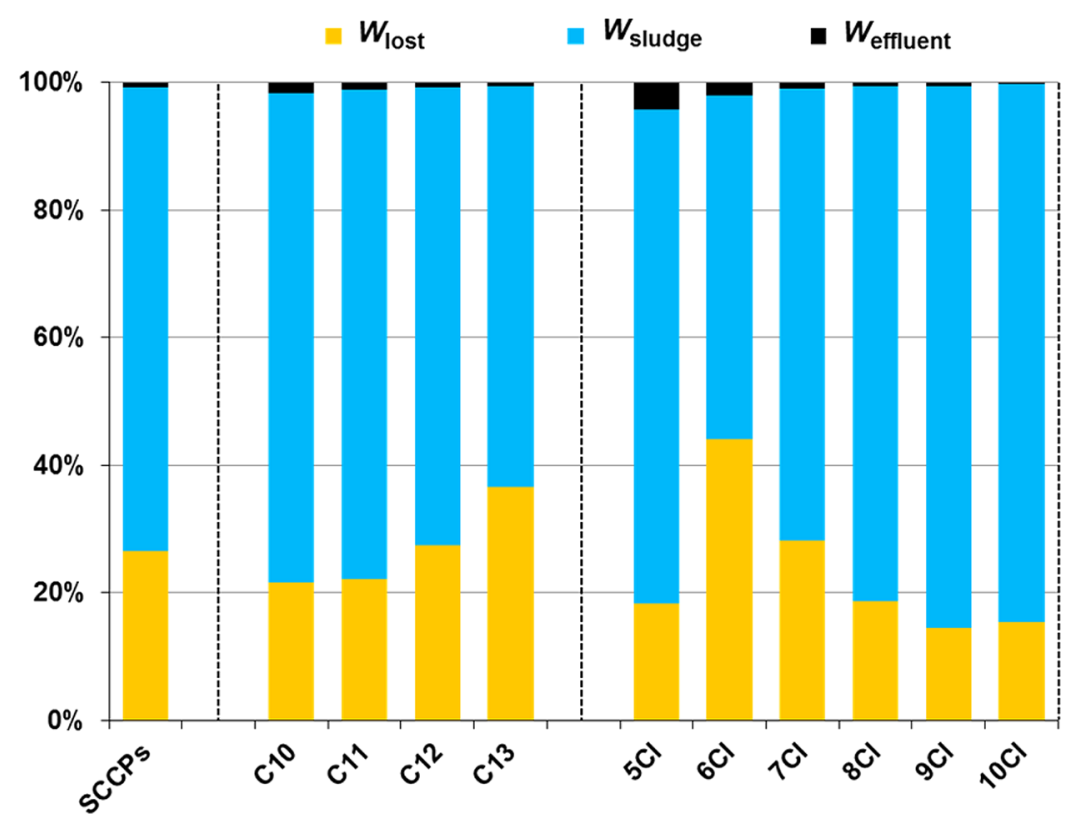

Figure 5. Mass balance results: mass fractions (\%) of detected $\sum$ SCCPs and different congener groups in (i) effluents $\left(W_{\text {efflent }}\right)$, (ii) dewatered sludge $\left(W_{\text {sludge }}\right)$, and (iii) total lost $\left(W_{\text {lost }}\right)$ relative to total initial mass loading $(100 \%)$ in the STP.

percentages were $12.6 \%, 7.2 \%$, and $18.5 \%$ in anaerobic, anoxic and aerobic treatment units, respectively. The efficiency of anaerobic and aerobic treatment seems to better than that of anoxic treatment. The total mass change percentages (combined aqueous phase with solid phase) were mainly dominated by the solid-phase mass change percentages and were observed at $4.1 \%, 0.2 \%$, and $1.1 \%$ in anaerobic, anoxic, and aerobic treatment units, respectively (Supporting Information, Figure S4). The low mass change percentages in the three secondary treatment units were due to their low degradation rate relative to high loading amount in activated sludge.

For the secondary effluent, the aqueous-phase mass flow of SCCPs were $7.3 \pm 0.6 \mathrm{~g} / \mathrm{d}$, and accounted for about $20 \%$ of initial mass flow in raw sewage, while the adsorbed amount could be ignored due to the low content of suspended solids in the effluent. The combination of primary and secondary treatments removed around $80 \%$ of the initial SCCP mass from the aqueous phase. It was suggested that, to a certain extent, activated sludge treatment was effective to remove SCCPs from sewage water by biodegradation and biosorption. The aqueousphase mass change percentage were about $8 \%$ in the secondary sedimentation tank, which might be related to the long hydraulic retention time $(8 \mathrm{~h})$ and partitioning equilibrium. The excess sludge was then moved through a dehydrator without further digestion, and total mass flow of SCCPs in dewatered sludge was $920 \pm 83 \mathrm{~g} / \mathrm{d}$ during the three sampling campaigns.

At the tertiary treatment stage, the mass flow after hyperfiltration showed a slight decrease because of the removal of little suspended solids. After ozonation process, the mass flow was further decreased, but the efficiency of SCCPs removal by ozonation is not so noticeable. Due to the relatively low mass loading in the secondary effluents, the mass change percentages during hyperfiltration and ozonation were also comparable to those in the secondary treatment units. In comparison, chlorination had no favorable effect on the removal of SCCPs. The slight increase of mass flow could be attributed to their potential variations during the three sampling days and the analytical deviation of low concentration. Although hyperfiltration, ozonation, and chlorination are useful for improving the removal of other organic pollutions in STPs, ${ }^{37,38}$ the efficacy for removing the remaining SCCPs in secondary effluent was found to be limited based on the results of this study.

The removal efficiencies in aqueous phase were calculated by comparing the dissolved mass in raw sewage and effluents. The removal efficiency for $\sum$ SCCPs in aqueous phase was $82.2 \%$ in this study. Additionally, the congener-specific removal efficiencies for $\mathrm{C} 10-\mathrm{C} 13$ homologues and $5 \mathrm{Cl}-10 \mathrm{Cl}$ homologues in aqueous phase ranged from 67.4 to $88.5 \%$ and from 45.5 to $100 \%$, respectively, which showed a corresponding increasing trend with the increase of carbon and chlorine atom numbers (Supporting Information, Figure S5). To further understand the congener-specific removal efficiencies, we calculated the apparent solid-water partition coefficients $\left(K_{\mathrm{d}}\right)$ of different homologues between sludge and sewage water. As shown in Table S4, the $K_{d}$ values significantly increased with increasing carbon and chlorine atom numbers, which were consistent with the variations of congener-specific removal efficiencies. On the other hand, the $K_{d}$ values also significantly increased along the aerobic-anoxic-anaerobic treatment process. These relationships suggested that sorption to sludge particles may be an important removal mechanism for the most hydrophobic SCCPs.

Mass Balance Analysis for Fate of SCCPs during Wastewater Treatment. Since only less than $1 \%$ of total SCCP mass entering the STP was finally detected in the effluent, the remaining mass must have undergone one or a combination of the following processes: volatilization, degradation, transformation, and sorption to sludge. To assess the contributions of various processes in the STP, mass balance calculation was performed to analyze the fate of SCCPs and their congener groups during wastewater treatment (Figure 5). We took total mass loading in raw sewage (including dissolved and suspended solid phases) as the system input (100\%), while the system output consisted of (i) secondary and tertiary 
effluents and (ii) dewatered sludge. The third part was expressed as (iii) lost, due to the total effect of degradation, transformation, and volatilization in each treatment unit in the STP. ${ }^{39}$

The mass fraction of $\sum$ SCCPs in dewatered sludge accounted for $72.6 \%$ of the initial loading, and only $0.8 \%$ of the total amount of SCCPs was found in the secondary and tertiary effluents, while $26.6 \%$ of them was lost probably due to biodegradation. Among four carbon chain groups, the mass fraction in dewatered sludge ranged from $62.9 \%$ to $76.8 \%$, which showed a gradual decreasing trend with increasing carbon chain. The mass fractions in effluents for $\mathrm{C} 10-\mathrm{C} 13$ homologues were between $0.5 \%$ and $1.7 \%$, and the variation of mass fraction was similar to that in dewatered sludge. The mass fractions in loss for $\mathrm{C} 10, \mathrm{C} 11, \mathrm{C} 12$, and $\mathrm{C} 13$ were $21.7 \%$, $22.1 \%, 27.5 \%$, and $36.6 \%$, respectively. This further confirmed that activated sludge treatments are more effective for removing longer chain congeners through biosorption and biodegradation/biotransformation during anaerobic-anoxic-aerobic treatment units.

Among six chlorine congener groups, the mass fractions in effluents decreased significantly from $4.2 \%$ for $5 \mathrm{Cl}$ to $0.2 \%$ for $10 \mathrm{Cl}$ with the increase of chlorine atoms, but in contrast, the mass fraction in dewatered sludge showed an apparent increasing trend with the increase of chlorine atoms except for $5 \mathrm{Cl}$ homologue. This could be explained that higher chlorinated congeners, the more hydrophobic components within SCCPs, could be easily adsorbed to activated sludge but difficultly degraded by microorganisms, resulting in that most of them were removed from the aqueous phase and finally incorporated into dewatered sludge. Due to the very small mass fraction in effluents, the mass fraction in loss for six chlorine congener groups were mainly dominated by the adsorbed mass fraction in sludge, which were $18.3 \%, 44.1 \%, 28.3 \%, 18.8 \%$, $14.5 \%$, and $15.4 \%$ in order of the increasing chlorine atoms.

It is noteworthy that the relatively high mass fractions of $\sum$ SCCPs and their congener groups were present in the final dewatered sludge. High mass accumulation of SCCPs in sludge could result from the long-term cycling in the STP, indicating that SCCPs are resistant to degradation and quite persistent in activated sludge. Therefore, the sludge is a huge reservoir for SCCPs during the wastewater treatment, which should be paid more attention for sludge management in the future.

\section{ASSOCIATED CONTENT}

\section{(S Supporting Information}

Detailed procedures on sample extraction and cleanup; quality assurance and quality control (QA/QC), water flow and regularly measured parameters in the STP (Table S1), detailed concentrations of chlorine congener groups in sewage water and sludge (Table S2), mass flux (g/d) of detected SCCP congener groups (Table S3), congener-specific solid-water partition coefficients $\left(K_{\mathrm{d}}\right)$ (Table S4), variations of $\sum$ SCCPs concentration in aqueous phase at various treatment stages (Figure S1), comparisons of SCCP congener group abundance profiles in sewage water and sludge samples at various treatment stages (Figure S2, S3), total mass change percentages of $\sum$ SCCPs at the A/A/O treatment units (Figure S4), and aqueous phase removal efficiency of different SCCP congener groups and $\sum$ SCCPs (Figure S5). This material is available free of charge via the Internet at http://pubs.acs.org.

\section{AUTHOR INFORMATION}

\section{Corresponding Author}

*Tel: 8610-6284-9334. Fax: 8610-6284-9339. E-mail: ywwang@rcees.ac.cn.

\section{Notes}

The authors declare no competing financial interest.

\section{ACKNOWLEDGMENTS}

This work was jointly supported by the National Natural Science Foundation (21007078, 21107122, 21222702, 20890111), the China Postdoctoral Science Foundation (2012M510052), and the President Fund of GUCAS (Y25101BY00). We also thank the State Key Laboratory of Environmental Chemistry and Ecotoxicology for supporting this work.

\section{REFERENCES}

(1) Eljarrat, E.; Barcelo, D. Quantitative analysis of polychlorinated $n$ alkanes in environmental samples. Trac-Trends Anal. Chem. 2006, 25 (4), 421-434.

(2) Santos, F. J.; Parera, J.; Galceran, M. T. Analysis of polychlorinated $n$-alkanes in environmental samples. Anal. Bioanal. Chem. 2006, 386 (4), 837-857.

(3) De Boer, J.; El-Sayed Ali, T.; Fiedler, H.; Legler, J.; Muir, D. C.; Nikiforov, V. A.; Tomy, G. T.; Tsunemi, K. Chlorinated Paraffins. In The Handbook of Environmental Chemistry, Chlorinated Paraffins; De Boer, J., Ed.; Springer-Verlag: Berlin/Heidelberg, 2010; Vol. 10.

(4) Reth, M.; Zencak, Z.; Oehme, M. First study of congener group patterns and concentrations of short- and medium-chain chlorinated paraffins in fish from the North and Baltic Sea. Chemosphere 2005, 58 (7), 847-854.

(5) Barber, J. L.; Sweetman, A. J.; Thomas, G. O.; Braekevelt, E.; Stern, G. A.; Jones, K. C. Spatial and temporal variability in air concentrations of short-chain $\left(\mathrm{C}_{10}-\mathrm{C}_{13}\right)$ and medium-chain $\left(\mathrm{C}_{14}-\mathrm{C}_{17}\right)$ chlorinated $n$-alkanes measured in the U.K. atmosphere. Environ. Sci. Technol. 2005, 39 (12), 4407-4415.

(6) Marvin, C. H.; Painter, S.; Tomy, G. T.; Stern, G. A.; Braekevelt, E.; Muir, D. C. G. Spatial and temporal trends in short-chain chlorinated paraffins in Lake Ontario sediments. Environ. Sci. Technol. 2003, 37 (20), 4561-4568.

(7) Bayen, S.; Obbard, J. P.; Thomas, G. O. Chlorinated paraffins: A review of analysis and environmental occurrence. Environ. Int. 2006, 32 (7), 915-929.

(8) Pellizzato, F.; Ricci, M.; Held, A.; Emons, H. Analysis of shortchain chlorinated paraffins: A discussion paper. J. Environ. Monit. 2007, 9 (9), 924-930.

(9) Friden, U. E.; McLachlan, M. S.; Berger, U. Chlorinated paraffins in indoor air and dust: Concentrations, congener patterns, and human exposure. Environ. Int. 2011, 37 (7), 1169-1174.

(10) Tomy, G. T.; Stern, G. A.; Lockhart, W. L.; Muir, D. C. G. Occurrence of $\mathrm{C}_{10}-\mathrm{C}_{13}$ polychlorinated $n$-alkanes in Canadian midlatitude and arctic lake sediments. Environ. Sci. Technol. 1999, 33 (17), 2858-2863.

(11) Tomy, G. T.; Muir, D. C. G.; Stern, G. A.; Westmore, J. B. Levels of $\mathrm{C}_{10}-\mathrm{C}_{13}$ polychloro- $n$-alkanes in marine mammals from the Arctic and the St. Lawrence River estuary. Environ. Sci. Technol. 2000, 34 (9), 1615-1619.

(12) Iozza, S.; Muller, C. E.; Schmid, P.; Bogdal, C.; Oehme, M. Historical profiles of chlorinated paraffins and polychlorinated biphenyls in a dated sediment core from Lake Thun (Switzerland). Environ. Sci. Technol. 2008, 42 (4), 1045-1050.

(13) Houde, M.; Muir, D. C. G.; Tomy, G. T.; Whittle, D. M.; Teixeira, C.; Moore, S. Bioaccumulation and trophic magnification of short- and medium-chain chlorinated paraffins in food webs from Lake Ontario and Lake Michigan. Environ. Sci. Technol. 2008, 42 (10), 3893-3899. 
(14) Cooley, H. M.; Fisk, A. T.; Wiens, S. C.; Tomy, G. T.; Evans, R. E.; Muir, D. C. G. Examination of the behavior and liver and thyroid histology of juvenile rainbow trout (Oncorhynchus mykiss) exposed to high dietary concentrations of $\mathrm{C}_{10^{-}}, \mathrm{C}_{11^{-}}, \mathrm{C}_{12^{-}}$, and $\mathrm{C}_{14^{-}}$-polychlorinated n-alkanes. Aquat. Toxicol. 2001, 54 (1-2), 81-99.

(15) Fisk, A. T.; Tomy, G. T.; Muir, D. C. G. Toxicity of C10-, C11-, C12-, and C14-polychlorinated alkanes to Japanese medaka (Oryzias latipes) embryos. Environ. Toxicol. Chem. 1999, 18 (12), 2894-2902.

(16) Persistent Organic Pollutants Review Committee. Supporting Document for the Draft Risk Profile on Short-Chained Chlorinated Paraffins, UNEP/POPS/POPRC.6/INF/15; Stockholm Convention on Persistent Organic Pollutants: Geneva, 2010.

(17) Zencak, Z.; Oehme, M. Recent developments in the analysis of chlorinated paraffins. Trac-Trends Anal. Chem. 2006, 25 (4), 310-317.

(18) Sverko, E.; Tomy, G. T.; Marvin, C. H.; Muir, D. C. G. Improving the quality of environmental measurements on short chain chlorinated paraffins to support global regulatory efforts. Environ. Sci. Technol. 2012, 46 (9), 4697-4698.

(19) Feo, M. L.; Eljarrat, E.; Barcelo, D. Occurrence, fate, and analysis of polychlorinated $n$-alkanes in the environment. Trac-Trends Anal. Chem. 2009, 28 (6), 778-791.

(20) Zeng, L. X.; Wang, T.; Ruan, T.; Liu, Q.; Wang, Y. W.; Jiang, G. B. Levels and distribution patterns of short chain chlorinated paraffins in sewage sludge of wastewater treatment plants in China. Environ. Pollut. 2012, 160, 88-94.

(21) Song, M.; Chu, S. G.; Letcher, R. J.; Seth, R. Fate, partitioning, and mass loading of polybrominated diphenyl ethers (PBDEs) during the treatment processing of municipal sewage. Environ. Sci. Technol. 2006, 40 (20), 6241-6246.

(22) Nakada, N.; Shinohara, H.; Murata, A.; Kiri, K.; Managaki, S.; Sato, N.; Takada, H. Removal of selected pharmaceuticals and personal care products (PPCPs) and endocrine-disrupting chemicals (EDCs) during sand filtration and ozonation at a municipal sewage treatment plant. Water Res. 2007, 41 (19), 4373-4382.

(23) Heidler, J.; Halden, R. U. Meta-analysis of mass balances examining chemical fate during wastewater treatment. Environ. Sci. Technol. 2008, 42 (17), 6324-6332.

(24) Schultz, M. M.; Higgins, C. P.; Huset, C. A.; Luthy, R. G.; Barofsky, D. F.; Field, J. A. Fluorochemical mass flows in a municipal wastewater treatment facility. Environ. Sci. Technol. 2006, 40 (23), $7350-7357$.

(25) Stevens, J. L.; Northcott, G. L.; Stern, G. A.; Tomy, G. T.; Jones, K. C. PAHs, PCBs, PCNs, organochlorine pesticides, synthetic musks, and polychlorinated $n$-alkanes in U.K. sewage sludge: Survey results and implications. Environ. Sci. Technol. 2003, 37 (3), 462-467.

(26) Iino, F.; Takasuga, T.; Senthilkumar, K.; Nakamura, N.; Nakanishi, J. Risk assessment of short-chain chlorinated paraffins in Japan based on the first market basket study and species sensitivity distributions. Environ. Sci. Technol. 2005, 39 (3), 859-866.

(27) Zeng, L. X.; Wang, T.; Wang, P.; Liu, Q.; Han, S. L.; Yuan, B.; Zhu, N. L.; Wang, Y. W.; Jiang, G. B. Distribution and trophic transfer of short-chain chlorinated paraffins in an aquatic ecosystem receiving effluents from a sewage treatment plant. Environ. Sci. Technol. 2011, 45 (13), 5529-5535.

(28) Zeng, L. X.; Wang, T.; Han, W. Y.; Yuan, B.; Liu, Q. A.; Wang, Y. W.; Jiang, G. B. Spatial and vertical distribution of short chain chlorinated paraffins in soils from wastewater irrigated farmlands. Environ. Sci. Technol. 2011, 45 (6), 2100-2106.

(29) Tomy, G. T.; Stern, G. A.; Muir, D. C. G.; Fisk, A. T.; Cymbalisty, C. D.; Westmore, J. B. Quantifying $\mathrm{C}_{10}-\mathrm{C}_{13}$ polychloroalkanes in environmental samples by high-resolution gas chromatography electron capture negative ion high resolution mass spectrometry. Anal. Chem. 1997, 69 (14), 2762-2771.

(30) Tomy, G. T.; Stern, G. A. Analysis of $\mathrm{C}_{14}-\mathrm{C}_{17}$ polychloro- $n$ alkanes in environmental matrixes by accelerated solvent extractionnigh-resolution gas chromatography/electron capture negative ion high-resolution mass spectrometry. Anal. Chem. 1999, 71 (21), 48604865.
(31) Tomy, G. T.; Westmore, J. B.; Stern, G. A.; Muir, D. C. G.; Fisk, A. T. Interlaboratory study on quantitative methods of analysis of C10C13 polychloro-n-alkanes. Anal. Chem. 1999, 71 (2), 446-451.

(32) Reth, M.; Oehme, M. Limitations of low resolution mass spectrometry in the electron capture negative ionization mode for the analysis of short- and medium-chain chlorinated paraffins. Anal. Bioanal. Chem. 2004, 378 (7), 1741-1747.

(33) Reth, M.; Zencak, Z.; Oehme, M. New quantification procedure for the analysis of chlorinated paraffins using electron capture negative ionization mass spectrometry. J. Chromatogr. A 2005, 1081 (2), 225231.

(34) Fan, Z. L.; Wu, S. M.; Chang, H.; Hu, J. Y. Behaviors of glucocorticoids, androgens, and progestogens in a municipal sewage treatment plant: Comparison to estrogens. Environ. Sci. Technol. 2011, 45 (7), 2725-2733.

(35) Liu, Y. S.; Ying, G. G.; Shareef, A.; Kookana, R. S. Occurrence and removal of benzotriazoles and ultraviolet filters in a municipal wastewater treatment plant. Environ. Pollut. 2012, 165, 225-232.

(36) Hilger, B.; Fromme, H.; Volkel, W.; Coelhan, M. Effects of chain length, chlorination degree, and structure on the octanol-water partition coefficients of polychlorinated $n$-alkanes. Environ. Sci. Technol. 2011, 45 (7), 2842-2849.

(37) Yang, X.; Flowers, R. C.; Weinberg, H. S.; Singer, P. C. Occurrence and removal of pharmaceuticals and personal care products (PPCPs) in an advanced wastewater reclamation plant. Water Res. 2011, 45 (16), 5218-5228.

(38) Hollender, J.; Zimmermann, S. G.; Koepke, S.; Krauss, M.; McArdell, C. S.; Ort, C.; Singer, H.; von Gunten, U.; Siegrist, H. Elimination of organic micropollutants in a municipal wastewater treatment plant upgraded with a full-scale post-ozonation followed by sand filtration. Environ. Sci. Technol. 2009, 43 (20), 7862-7869.

(39) Jia, A.; Wan, Y.; Xiao, Y.; Hu, J. Y. Occurrence and fate of quinolone and fluoroquinolone antibiotics in a municipal sewage treatment plant. Water Res. 2012, 46 (2), 387-394. 